\title{
EL DISCURSO DEL SUMAK KAWSAY Y EL GRAN SALTO INDUSTRIAL EN BOLIVIA
}

\section{Breve análisis del desarrollo rural del programa nacional de gobierno (2010-2015)}

\author{
Otto Colpari \\ Facultad Latinoamericana de Ciencias Sociales, FLACSO-Ecuador
}

Resumen: El partido MAS-IPSP del gobierno boliviano de Evo Morales Ayma en su última campaña electoral presentó un Programa Nacional de Gobierno (2010-2015) que delinea los principales elementos para las futuras políticas públicas de Bolivia. El interés del presente trabajo es develar cual es enfoque del desarrollo rural de este programa en un contexto del discursivo del Sumak Kawsay o el Buen Vivir.

Palabras clave: Sumak Kawsay, industrialización, desarrollo rural

Abstract: The MAS-IPSP movement of Bolivian government of Evo Morales Ayma in the last electioneering, showed a National Government Program (2010 -2015) which outlines the key elements for future public policy in Bolivia. The goal of this study is to identify the approach of rural development of this program in a context Sumak Kawsay ("Good Life") discursive.

Keywords: Sumak Kawsay, industry, rural development

\section{Introducción}

El economista francés Serge Latouche (2007) sostiene que antes del contacto con occidente, en muchas civilizaciones (sino en todas) el concepto de desarrollo se encontraba ausente. En ese sentido, el desarrollo es un concepto vinculado con la historia de occidente, "y el desarrollo constituye a la vez una impostura conceptual, por su pretensión universalista, y una impostura práctica, por sus contradicciones profundas" (Latouche, 2007:53).

El desarrollo al ser una creencia occidental empezó a ser cuestionada en distintos ámbitos, ya sea en la academia, en la política, en los distintos movimientos ecologistas, en los sucesivos discursos de la "izquierda", etc. Específicamente aquí nos interesa el llamativo ascenso de un discurso en contra del desarrollo capitalista que se ha ido esbozando en los gobiernos de América Latina. Particularmente son importantes en esa línea las posturas de Bolivia y Ecuador, que por primera vez en su historia han entrado en un "debate público" sobre su desarrollo y la forma de organizar este, con sus nuevas constituciones (Bolivia, 2007 y Ecuador, 2008) incorporaron el concepto 
del Vivir Bien o Sumak Kawsay como un eje articulador de una propuesta de desarrollo alternativo al concepto occidental de desarrollo.

El Sumak Kawsay, el Buen Vivir o el Vivir Bien es un concepto tomado de uno de los principales movimientos organizados contra las políticas del ajuste estructural aplicadas hacia dos décadas atrás, me refiero al movimiento indígena, campesino y originario de Ecuador y Bolivia. Ese espíritu de transformación del movimiento indígena ha sido tomado por algunos académicos (Acosta, 2009, Dávalos, 2008; Medina, 2006) para afirmar que el Sumak Kawsay es una noción para dejar atrás el concepto clásico de desarrollo y subdesarrollo impuesto después de la Segunda Guerra Mundial para caracterizar aquellos países modernizados de los atrasados.

En esa línea Bolivia y Ecuador han tomado como una política estatal la consecución del Sumak Kawsay, por un lado en el Ecuador se expresa claramente esta política en su Plan Nacional Para el Buen Vivir (2009-2013). El Buen Vivir según el gobierno ecuatoriano busca una "ruptura conceptual (...) con los idearios del Consenso de Washington y con las aproximaciones más ortodoxas del concepto de desarrollo" (PNBV, p. 5). En el caso de Bolivia en la campaña electoral del presidente boliviano Evo Morales su partido el MASIPSP presentó su Programa de Gobierno, Bolivia Líder ${ }^{1}$ (2010-2015), donde se proclama una Bolivia símbolo en el mundo que cuestiona "el modelo de acumulación capitalista y los patrones de consumo de la civilización occidental" (MAS-IPSP, 2009:146) es por eso que su propuesta de gobierno está orientada a forjar "un mundo justo, diverso, inclusivo, equilibrado y armónico con la naturaleza para el 'Vivir Bien' de todos los pueblos a nivel mundial" (MASIPSP, 2009:56).

Sin embargo, es necesario revisar que implica el Sumak Kawsay en las prácticas concretas que realizan los precursores y defensores de esta noción de desarrollo. He querido centrar mi análisis precisamente en aquellos actores que iniciaron la consecución de esta noción, es decir los actores y sujetos del mundo rural. Para intentar hacer este análisis elegimos a Bolivia donde la pobreza extrema en el área rural, según los datos del Instituto Nacional de Estadística bajó de $62.9 \%$ en 2005 a $49.2 \%$ en 2008. En ese contexto surge la pregunta de ¿cuál es el enfoque del "desarrollo rural en el reciente plan de gobierno boliviano (2010-2015) en un contexto discursivo de la construcción del Sumak Kawsay o el Buen Vivir?.

Para ello vamos dividir el presente ensayo en dos partes primero haciendo un análisis del Plan de Gobierno del presidente Evo Morales, que básicamente está centrada en la industrialización. En una segunda parte veremos el papel que tiene el desarrollo rural en este escenario del crecimiento económico mediante un proceso de industrialización. Para concluir con el papel que asume el Sumak Kawsay en la lógica de los anteriores puntos.

\footnotetext{
${ }^{1}$ En adelante utilizaremos la sigla MAS-IPSP para referirnos a dicho programa
} 


\section{Bolivia hacia un "Gran Salto Industrial"}

En el manifiesto no comunista de Rostow (1961) que plantea las etapas para un crecimiento económico nos sugiere que una etapa necesaria (un impulso inicial) para el crecimiento económico de un país es de índole tecnológica. "El impulso inicial tuvo que esperar la formación de capital social fijo y una oleada de desarrollo tecnológico en la agricultura y la industria (...) durante el impulso inicial nuevas industrias se expanden con rapidez produciendo utilidades" (Rostow, 1961:30). Este impulso inicial al parecer está presente como una de las propuestas principales del gobierno de Evo Morales.

El programa de gobierno propuesto por el Movimiento al Socialismo (MAS)2 para los años 2010-2015, se basa fundamentalmente en dos modelos económicos, por un lado la industrialización como base de un nuevo modelo productivo: el "Gran Salto Industrial"; y por el otro lado, con menor énfasis un modelo de economía plural (pública, mixta y privada) que permitiría dinamizar el desarrollo del conjunto de los sectores productivos del país. En síntesis, la propuesta del gobierno boliviano es lograr un tránsito de un modelo predominante liberal y neoliberal de desarrollo, basado en un patrón primario exportador excluyente, hacia "otro modelo económico que se caracteriza por impulsar procesos de industrialización de los recursos naturales y de agregación de valor" (MAS-IPSP, 2009:109).

Se podría decir que en Bolivia se quiere iniciar un proceso que en algunos países de América Latina comenzó con "la depresión mundial de los treintas [que] derribó los fundamentos del modelo de exportaciones primarias y arrojó a la América Latina a una intensa sustitución de importaciones como respuesta al desplome de los ingresos por exportaciones" (Sheanan, 1990:115). Desde ese punto de vista, el plan nacional de desarrollo de Bolivia, se asemeja mucho a la aplicación de la teoría estructuralista cepalina de los años 60, que buscaba el desarrollo mediante la planificación económica y la industrialización como punta de lanza apoyada en la industrialización por sustitución de importaciones (ISI). Este programa del MAS-IPSP esboza y caracteriza con mucha precisión las áreas hacer industrializadas como son los hidrocarburos, minerales, alimentos, producción de medicamentos, textiles y, en general, toda aquella actividad que produzca valor agregado.

En síntesis, dar el "Gran Salto Industrial" significa cumplir con una tarea largamente postergada. Industrializar nuestros recursos naturales significa crear una matriz económica que genera valor agregado, empleo y mayores recursos económicos para atender las demandas de los sectores menos favorecidos por los viejos modelos que convirtieron a nuestro país en uno de los más pobres del continente. Partiendo del "Gran Salto Industrial" como una base fundamental del Programa de Gobierno 2010-2015. (MAS-IPSP, 2009:74)

Es así que en Bolivia se esta lejos de apostar al "buen vivir que es una concepción de la vida alejada de los parámetros más caros de la modernidad y

\footnotetext{
${ }^{2}$ Partido político del actual presidente boliviano Evo Morales Ayma.
} 
el crecimiento económico" (Dávalos, 2008). En Bolivia el Sumak Kawsay parece aplicarse entre las estrategias de desarrollo planteadas por la teoría estructuralista de la CEPAL y por la teoría de la modernización. En el caso de la visión estructuralista según Cristobal Kay (2007) se tenía una ideología antifeudal, antioligarquica reformista y tecnocrática, características que actualmente propugnan en el gobierno de Bolivia. Para Kay el proceso estructuralista "conllevaba un aumento de los gastos gubernamentales dedicados a promover el desarrollo, pero fue incluso más lejos, ya que contemplaba al Estado como el agente crucial en el cambio económico, social y político" (2007:54). Es por esta razón que para la aplicación de este nuevo modelo productivo en Bolivia, se pretende poner énfasis en las siguientes estrategias: a) expansión del rol del Estado, b) desarrollo productivo con valor agregado de los recursos naturales y sustentabilidad ambiental, c) participación activa de las economías (pública, privada, comunitaria y cooperativa) en el aparato productivo, d) producción para satisfacer el mercado interno y luego la exportación, y c) redistribución equitativa de la riqueza y excedentes y reducción de las desigualdades. (MAS-IPSP, 2009:109-110).

También asumimos que Bolivia busca su inclusión en el proyecto de la modernidad porque se tiene la premisa de que el capital, la ciencia y la tecnología tienen efectos beneficiosos para la sociedad. Se asume que el bienestar deviene de la industrialización y el uso adecuado de la tecnología. Una reproducción de la sociología del desarrollo que receta que "los países del Tercer Mundo deberían seguir la misma senda que los países capitalistas (...) los países ricos desarrollados difundirían conocimientos, capacidades, tecnología, organización, instituciones, actitudes empresariales y espíritu innovador entre las naciones pobres del sur" (Kay, 2007:52)

A esta serie de medidas de potenciamiento de la producción, nuestro Programa de Gobierno la llama el "Gran Salto Industrial"; una iniciativa vital postergada durante décadas y hasta siglos. Hoy, gracias a la diversificación de la tecnología, que la hace más accesible, y considerando el buen momento económico que atraviesa Bolivia, superados sus déficit fiscales y con un nivel histórico de reservas internacionales, Bolivia puede invertir en un proceso de industrialización a gran escala. Para ello, es posible que se requiera el concurso de nuevos y mayores capitales en calidad de socios estratégicos pero bajo el principio del control estatal, tal como ha venido ocurriendo en estos tres años y medio de proceso de cambio (MAS-IPSP, 2009:74)

De esta manera la industrialización se convierte en el pilar fundamental de desarrollo de Bolivia y es el "paraguas" para el "desarrollo" del mundo rural y del Sumak Kawsay - un concepto que parecía radicalmente distinto al occidental- esto trae a colación un debate que los economistas han tenido por bastante tiempo entre aquellos que creen que la agricultura es el motor del desarrollo los llamados agraristas y aquellos que suponen que con la industria resolveremos todos nuestros problemas los llamados industrialistas (Kay, 2009). En ese sentido el plan nacional del gobierno de Bolivia (2010-2015), no esclarece una postura intermedia o complementaria sino apuesta por la vía de los Ilamados industrialistas que seria principal factor de eliminación de la 
pobreza, basta con observar que casi la totalidad de la propuesta económica del gobierno se basa en esa premisa de este tipo.

Es por ello que advertimos que el programa del MAS-IPSP no tiene claramente dilucidado las conexiones entre la industria y agricultura, olvidando la importante relación de la industria con una revolución agrícola y las nuevas actividades no agrícolas del mundo rural. Un planificador económico como Lewis nos diría que si la pretensión del Estado boliviano es el progreso mediante el crecimiento económico y por medio del aumento de la tecnología para mejorar la calidad de vida de los bolivianos y bolivianos es importante que "la industrialización dependa de las mejoras en la agricultura, que no es rentable producir un creciente número de manufacturas a menos que la producción agrícola crezca de modo simultáneo. Esa es la razón que explica por qué las revoluciones industriales y agrícolas van siempre juntas, y por qué en las economías en las cuales la agricultura se estanca, tampoco hay desarrollo industrial (En: Kay, 2009:15). De acuerdo al plan de gobierno se crearan industrias que produzcan una mejora significativa en la agricultura como son la producción de fertilizantes minerales como el amoniaco y la urea pero no se especifica los nexos de sostenimiento de la agricultura, esto es importante para que esta no se vuelva una subsidiaria de la industrialización.

Es importante también que el "Gran Salto Industrial" tome en cuenta el enfoque de la nueva ruralidad, que se centra en el estudio en las actividades rurales fuera de la granja, como es la flexibilización y feminización del trabajo rural, las interacciones entre el ámbito urbano y rural: "rurarbanizacion", la migración y el papel de las remesas, etc.

"Una de las contribuciones del análisis de la nueva ruralidad es el cuestionamiento de la presunción de muchos analistas y encargados de formular las políticas públicas, de que las comunidades rurales están muy bien integradas en los mercados y que no operan exclusivamente dentro de una lógica de agricultura de subsistencia. La evidencia presentada por los nuevos ruralistas revela que los campesinos desarrollan múltiples actividades (es decir, pluriactividad y multifuncionalidad agrícolas y no agrícolas, dentro y fuera de la granja, y que también son productores y jornaleros asalariados. Por tanto, los campesinos se insertan en una variedad de mercados y cuentan con muchos nexos con las zonas urbanas. El estudio de estos cambios no se limita al ámbito económico, aunque se le da prioridad, sino que también abarca cambios en la sociedad, en especial aquellos relacionados con el surgimiento de nuevos actores y movimientos sociales" (Kay, 2009b:613).

Esta perspectiva de la nueva ruralidad daría en cuenta de la inclusión de las nuevas formas de organización económica que han surgido a través del desarrollo del capitalismo, como son aquellas relacionadas con el desarrollo económico local y serian importantes para la identificación de los vínculos con la industria. 
Este modelo de correlación entre industria y agricultura ha estado presente en la experiencias de dos de los nuevos países industriales del Asia del Este: Corea del Sur y Taiwan, para Kay (2009b) el éxito de estas actividades es una "estrategia de desarrollo sinérgica", donde el Estado tenga un papel primordial para crear vínculos entre la agricultura y la industria, garantizando incrementos sostenidos en eficiencia tanto en agricultura como en la industria. Acompañada de una profunda reforma agraria que contribuya a la generación de una estructura rural conducente al crecimiento y la equidad. En esa vía el programa de gobierno del MAS-IPSP canaliza una interesante expansión del Estado para la aplicación de este modelo nacional productivo:

Los roles del Estado se fortalecen y se complementan con las fuerzas del mercado, y además se moviliza a las instituciones de productores (asociaciones, sindicatos, comunidades, ayllus y otras organizaciones económicas) potenciándolos en su rol de proveedores y productores de bienes y servicios públicos, a través del desarrollo de mecanismos de acción colectiva organizada. En este sentido, se supera la tradicional dicotomía que ha existido en Bolivia entre Estado y mercado; dicotomía que condicionó la vida del país durante el último siglo (MAS-IPSP, 2009:87)

En consecuencia esta armazón planificadora parece apuntar a un desarrollo rural encausado en el tren de la industrialización bajo la retórica del Sumak Kawsay, en ese sentido es importante para el desarrollo rural tomar en cuenta la inclusión de Bolivia al mercado global y los distintos cambios que se producen en este. El hecho de que el MAS-IPSP reproduzca un sistema de producción del cual es difícil de superar no deja de lado la responsabilidad que se tiene en cuanto a la reducción de la pobreza del área rural y la competencia que tiene los pequeños productores frente a los campesinos capitalistas y agroexportadoras.

Así, la historia se repite, el progreso occidental "se muestra como una necesidad natural que nada podrá detener: el desarrollo no es una opción, sino la finalidad $-y$ la fatalidad- de la historia (Rist, 2002). La industria se vuelve bella y única arma para el bienestar y queda muy lejos de concretarse una verdadera relación armónica con la madre tierra. Parecen alejarse de Bolivia propuestas alternativas de desarrollo como el Sumak Kawsay, el posdesarrollo (Escobar, 2005), el decrecimiento (Latouche), las islas de sustentabilidad (Bebbigton) la economía social y solidaria (Coraggio) -incluso presente en la actual propuesta pero subordinada a la gran industria-, tal vez simplemente lo que pasa en Bolivia es la aplicación de los análisis marxistas para solucionar las contradicciones que produce el capitalismo ya que para la tradición ortodoxa del marxismo "el único remedio una vez terminada la revolución, no es más que reforzar la acumulación de capital, es decir del desarrollo" (Latouche, 2003:53)

Ésta es la razón por la que el "desarrollo" presentado siempre como una solución, es, en realidad un problema. Para utilizar una comparación, los "actuales gestores del desarrollo" se parecen a los antiguos alquimistas que intentaron vanamente convertir el oro en plomo, convencidos de que 
dispondrían así del secreto de la riqueza. Desaparecidos los alquimistas pareció que la autentica riqueza venia de otro sitio, es decir, de los hombres y del comercio. ¿Cuándo caeremos en la cuenta de que el bienestar no procede del crecimiento? (Rist, 2002)

\section{Bolivia: ¿En equilibrio con la madre tierra?}

Para Cristóbal Kay "el mundo atraviesa un periodo de cambio histórico: por primera vez en la historia de la humanidad, la población urbana excede a la del campo" (2009b:1). También esta constatación sucede en el territorio boliviano que de acuerdo a los datos de Urioste y Kay (2005) "al momento de la independencia en 1825, Bolivia tenía aproximadamente 11 mil comunidades registradas. Un siglo más tarde quedaron solamente 3.783 comunidades como resultado de los efectos devastadores del régimen latifundista feudal impuesto por los criollos" (23). En la actualidad en Bolivia "se observa un creciente proceso de urbanización que determina una disminución progresiva de la participación de la población rural en la población total, que actualmente llega a un 34\%" (Baudoin, 2009:3).

CUADRO 1: DATOS GENERALES POBLACIONALES

\begin{tabular}{||l|r|r||}
\hline \hline & POBLACIÓN & PARTICIPACIÓN \\
\hline Población (millones de personas) & 10.227 .299 & 100 \\
\hline Población URBANA (millones de personas) & 6.790 .084 & 66 \\
\hline Población RURAL (millones de personas) & 3.437 .215 & 34 \\
\hline \multicolumn{2}{|l}{ Fuente: Instituto Nacional de Estadística (INE) }
\end{tabular}

En: Baudoin, 2009

Estos cambios demográficos son para "algunos autores [un] proceso de desagrarizacion del campo (Bryceson, 2000; Escalante et al., 2007) otros más recientes hablan del surgimiento de las familias rurales postagrícolas (Grammont y Arias, 2008" (En Kay, 2009:614-615). En el estudio realizado por Luis F. Baudoin (2009) sobre la población rural en Bolivia se comprueba que dentro las principales actividades productivas, "predomina claramente el sector terciario de la economía (comercio y servicios), por otro, un rezago importante de la actividad secundaria y aún peor de la actividad primaria (solamente agropecuaria)" (p. 7) 


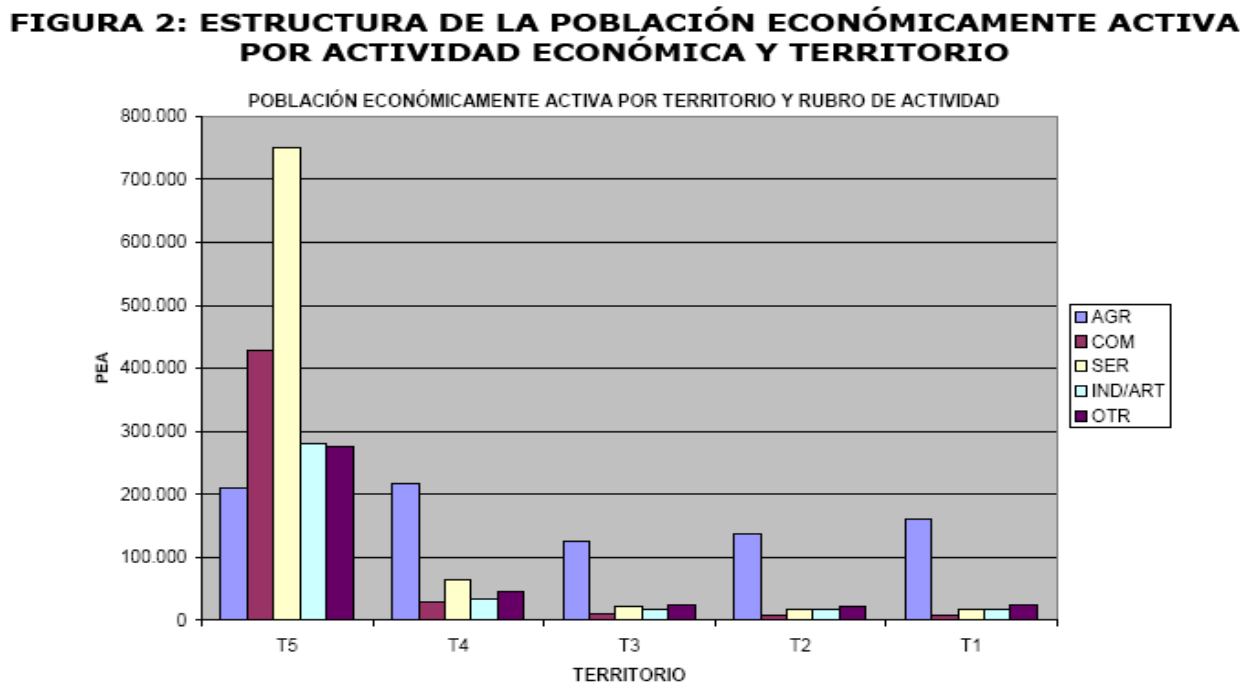

Fuente: Baudoin, 2009

Estos cambios demográficos y de actividades productivas no se encuentran presentes en el programa de gobierno boliviano (2010-2015), se pone bastante énfasis en el desarrollo rural mediante la ejecución de los siguientes principios: modificación de la tenencia y acceso a la tierra, cambio de los patrones productivos y alimentarios, creación de ingresos y excedentes agropecuarios forestales y por último la consolidación de una normatividad para la producción agrícola. Probablemente estos principios hacen que los planificadores de Bolivia caigan en el error de que el mundo rural boliviano es predominantemente agrícola, forestal y ganadero. Por ello Kay (2009) promueve la necesidad de tomar en cuenta los análisis de la nueva ruralidad en los estudios rurales:

Los nuevos ruralistas criticaron a los responsables de formular las políticas y a las organizaciones no gubernamentales por apegarse a un enfoque tradicional "productivista" hacia el desarrollo rural que por prestar demasiada atención a la agricultura, la ganadería y la producción forestal, no percibía la naciente realidad y los nuevos desafíos que afrontan los agricultores, los campesinos y los trabajadores del campo. (Kay, 2009:621)

Bolivia heredo un modelo de desarrollo rural basado en la premisa de "exportar o morir" privilegiando al sector agroexportador y la producción de los cultivos industriales, sin incentivar a la producción de alimentos para el mercado nacional, y en algunos casos creando dependencia extrema, tal es el caso del trigo. Para Baudoin (2009), Bolivia "necesita ganar espacio en seguridad alimentaria para evitar ser arrastrada por la depresión y la especulación del mercado internacional. Se requiere producir de manera acelerada alimentos básicos (trigo, maíz, papa, quinua, arroz, carnes y leche)" (p.39). Para ello el plan nacional de una "Bolivia Líder" propone un nuevo impulso a las políticas agrarias y medioambientales que ha venido implementando el gobierno nacional, veamos a continuación los puntos sobresalientes al respecto.

El gobierno desde el año 2005 está impulsando la consolidación de la propiedad comunal de la tierra, para ello en primera instancia se ha propuesto 
"eliminar el latifundio (latifundio cero) y recuperar tierras fiscales para la nación: Desarrollar un saneamiento de la propiedad agraria para la consolidación de las propiedades rurales trabajadas y a la reversión de las tierras ociosas que no cumplen con la función económico-social" (MAS-IPSP, 2009).

Esta política estatal va en contra de las políticas neoliberales aplicadas en anteriores gobiernos que abandonaban "la centralidad que los estructuralistas habían otorgado a la expropiación y la [sustituyen] por un énfasis en la privatización, la des-colectivización y el registro y la titulación de tierra" (Kay, 2007:75). Sin embargo, hay que reconocer que la lógica del saneamiento de tierras, sigue respondiendo a una lógica neoliberal del desarrollo rural que propicia la creación de un mercado de tierras para asegurar los títulos de propiedad, los neoliberales señalan "que los derechos de propiedad seguros y transparentes facilitarán las transacciones de tierra y darían a los productores el acceso al crédito en el mercado financiero formal dado que iban a utilizar su propiedad como garantía" (Kay, 1998:88). Claro que la diferencia fundamental en este proceso es la consolidación de una propiedad comunal frente a la propiedad individual, la colectivización de la tierra se lograría mediante la "aplicación de las disposiciones de la Ley 3545 de Reconducción Comunitaria y el resultado del Referéndum Constitucional sobre la extensión máxima de la propiedad, revirtiendo las tierras en desuso, mal utilizadas y excedentes" (MASIPSP, 2009:114)

Este tipo de reforma agraria tiene un riesgo latente a mi consideración pues conllevaría a legalizar las grandes propiedades agroexportadoras - muchas de ellas conseguidas por redes de clientelismo en la época de la dictadura - que generalmente depende de capitales extranjeros y que serian un potencial competidor de los pequeños y medianos productores en el mercado. Esto imposibilitaría un desarrollo sostenido del sector de la llamada economía plural, que se basan básicamente en estas pequeñas unidades domesticas de producción. En ese sentido las reflexiones históricas de Cristóbal Kay (1998) sobre la influencia de las empresas capitalistas que tienen grandes extensiones sobre el carácter colectivista del sector reformado son importantes:

Aunque los beneficios potenciales de derechos de propiedad claramente definidos pueden ser considerables dada la carencia de títulos de propiedad (...) el contexto económico y sociopolítico bajo el cual los pequeños agricultores están operando conspira en su contra. Hasta ahora la evidencia señala que todo lo que se logra es "modernizar la inseguridad (...) tiene que reconocerse que los títulos de propiedad que se basan en la costumbre así como los arreglos de arrendamiento en las comunidades rurales a menudo ofrecen mayor seguridad y flexibilidad a los campesinos que los esquemas de títulos de tierras del tipo del Banco Mundial (Kay, 1998:92)

La lógica del saneamiento y titulación de tierras aplicada desde el Estado boliviano tiene por finalidad corregir las distorsiones técnicas y jurídicas de la propiedad de la tierra para continuar redistribuyendo tierra a los campesinos y población indígena que no poseen tierra alguna o solamente una parcela insuficiente. De acuerdo al plan nacional de gobierno de Bolivia, se lograron 
titular 26 millones de hectáreas, beneficiando a 98.454 familias en los departamentos de La Paz, Santa Cruz, Tarija, Beni y Pando, gracias a la promulgación de la Ley de Reconducción Comunitaria de la Ley INRA (2006), conjuntamente con la Implementación del Plan Nacional de Saneamiento y Titulación de la Propiedad Agraria y el Plan Nacional de Distribución de Tierras y Asentamientos Humanos.

Para autores como Kay (2007) la reforma agraria es una medida necesaria pero insuficiente, para este autor la experiencia de las reformas agrarias durante la segunda mitad del siglo pasado han demostrado "que para que una reforma agraria reduzca la pobreza y aumente la equidad, es necesario que el Estado diseñe una serie de medidas de apoyo para los beneficiarios (...) entres estas figuran el suministro de asistencia técnica, crédito y facilidades de comercialización". EI MAS-IPSP en el programa de gobierno analizado plantea apoyar a la agricultura mediante un Seguro Agrícola Universal como parte de las políticas de incentivo a la producción y a la seguridad con soberanía alimentaria

\begin{abstract}
"Nuestro Programa de Gobierno propone implementar una serie de instrumentos y mecanismos que permitan el acceso, y la estabilización financiera y productiva del sector agrícola, así como la reducción de riesgos en la producción y comercialización de sus productos (...) el Seguro Agrícola cubrirá a las 650.000 unidades productivas grandes, medianas y pequeñas que existen en el país y que representan una superficie de 2.046.335 hectáreas cultivadas. De éstas, el $53.47 \%$ (1.094.257) corresponden al cultivo de oleaginosas; el 37.74\% (772.389) a cereales y el $8.52 \%$ a quinua, papa y otros. (MAS-IPSP, 2009:106)
\end{abstract}

Por otra parte, con la dotación de tierras fiscales "se han beneficiado 4.682 familias en todo el país. En tres años y medio de Gobierno se distribuyeron 958.064 hectáreas de tierras más que los gobiernos anteriores" (MAS-IPSP, 2009:26). De acuerdo a la planificación el proceso de saneamiento debe concluir hasta el año 2013 acompañado de una "dotación colectiva y planificada a comunidades campesino-indígenas"

La colectivización de la tierra se basará en un programa de reconstitución territorial, reconstituir territorios de pueblos indígenas por la vía de la expropiación de área a las cuales han tenido acceso y que son necesarias para su reproducción cultural y económica [seguido de un] plan de servidumbre cero: para acabar con las formas de servidumbre que vulnerando los derechos y las libertades humanas esenciales, todavía afectan a numerosas comunidades indígenas y campesinas en el área rural del país y, especialmente, en los territorios guaranís del Chaco (MAS-IPSP, 2009:114)

Estas políticas estatales de Bolivia aportan teóricamente al debate de la nueva ruralidad entorno a un enfoque de corte comunitario o territorial, en el caso del enfoque comunitario, tal vez encontraríamos algunos elementos iniciales para pensar el Suma Kawsay 
El enfoque territorial, por lo menos en su interpretación más popular, busca integrar más estrechamente a los pequeños agricultores con el mercado mundial, mientras que en la perspectiva comunitaria se hace hincapié en la autosuficiencia y la autonomía. Por otra parte, para lograr esta integración, el enfoque territorial propone incorporar a los minifundistas en las asociaciones agroindustriales para favorecer su transformación productiva y mejorar su competitividad. Esto requeriría crear alianzas entre las compañías transnacionales, los agricultores capitalistas y los campesinos, lo que representa una importante diferencia del enfoque comunitario en el que las empresas transnacionales y los capitalistas rurales son vistos como parte del problema, más que como solución para la pobreza rural. (Kay, 2009b:631)

Sin embargo, como hemos visto en el programa de gobierno del presidente Morales apunta a un modelo económico basado en la industrialización que hace sugerir que el papel del desarrollo rural y la agricultura pase por la necesaria inclusión de sus productores al mercado mundial. Por ello es que una de las propuestas industrializadoras en el mundo rural es la producción de "fertilizantes minerales y la fertilización agrícola, [para] incrementar tanto la productividad como mejorar la calidad de los productos agrícolas bolivianos" (MAS-IPSP, 2009:77) sin estas políticas para la industria agrícola significaría un "retraso en la competitividad del sector agrícola boliviano" (MAS-IPSP, 2009:G78). En ese marco de la industrialización es que se piensa las políticas del área rural se impulsará, vale decir que el desarrollo rural en Bolivia estará bajo el "paraguas" del incentivo a la industrialización, es así que se busca fortalecer las capacidades en los procesos de producción, industrialización y comercialización de productos estratégicos con las organizaciones productivas campesinas e indígenas comunitarias:

\section{Conclusión}

Pimentel (1977) calculó que si la población mundial se alimentará con una dieta como la de los norteamericanos y producida en las mismas condiciones, las reservas totales de petróleo del mundo se agotarían en solo tres años con sólo ese uso" (En: Warman, 1988:213).

De acuerdo a Latouche (2003) si seguimos la senda del desarrollo de occidente necesitaríamos seis planetas para vivir con la calidad de vida de occidente, pues el planeta se hace insostenible. El Sumak Kawsay de esta manera pierde sustancia en las políticas aplicadas por el primer presidente indígena de Bolivia, valdría la pena preguntarse si el objetivo y las luchas del movimiento indígena originario y campesino están orientados a la modernización del Estado. Es identificable esta perspectiva en las luchas contra el colonialismo interno y el carácter colonial del Estado fueron para una mayor inclusión y participación en la toma de decisiones, que para algunos están encaminadas en la lógica de la modernización. En la actualidad ¿las luchas por el territorio y la tierra en los países andinos se convertirán en un precursores de la salida del atraso por una modernidad esperada?. 
Arturo Escobar (2005) en uno de sus artículos de postdesarrollo decía "si aceptamos ya sea la necesidad de rebasar la modernidad o el planteamiento de que estamos en un periodo de transición paradigmática, esto quiere decir que los conceptos de desarrollo y Tercer Mundo ya pertenecen al pasado" (p.31). En Bolivia si bien ha cambiado la utilización de conceptos y nociones las prácticas de un discurso del desarrollo occidental al menos en las manos del partido MAS-IPSP continúa, confundiendo el aumento del crecimiento económico con el aumento de la calidad de vida. El gobierno de Bolivia esta más en concordancia con el informe del Banco Mundial (BM) del año 2008 que con propuestas alternativas al capitalismo. De acuerdo a este informe que está dedicado íntegramente a la agricultura se habla de la existencia de "tres mundos de la agricultura": los países agrícolas, los países en proceso de transformación y los países urbanizados, en este última categorización se encuentra Bolivia y los países de América Latina, para ellos el BM recomienda como objetivo vincular a los pequeños agricultores con los mercados de alimentos y generar empleos remunerativos en las zonas rurales.

El Gran Salto industrial de todos modos trata de dar un mayor papel al Estado boliviano que busca mediante esta vía solucionar los problemas de pobreza en el país. Acompañada de una importante reforma agraria que en principio trata de reconstruir los territorios indígenas dotando estos de manera colectiva, que puede ser un principio de un enfoque comunitario (Barkin, 2001) o del Sumak Kawsay.

\section{Bibliografía}

ACOSTA, Alberto (2009), "El Buen Vivir, una utopía por (re)construir" en Revista Casa de las Américas, № 257, La Habana, octubre-diciembre del 2009.

BAUDOIN, L. F. (2009). "Crisis y pobreza rural en América Latina: el caso de Bolivia". Documento de Trabajo $\mathrm{N}^{\circ}$ 40. Programa Dinámicas Territoriales Rurales. Rimisp, Santiago, Chile.

BARKIN, David, comp. (2001) Innovaciones Mexicanas en el Manejo del Agua. México: Centro de Ecología y Desarrollo y Universidad Autónoma Metropolitana-Xochimilco.

BANCO MUNDIAL (2008), Informe sobre el desarrollo mundial 2008: Agricultura para el desarrollo, Bogotá, D.E., 2008.pp. 301.

DÁVALOS, Pablo. (2008) El "Sumak Kawsay" ("Buen vivir") y las censuras del desarrollo. En: Asamblea Constituyente. [en línea]. [consultado 31 jul. 2008]. Disponible en <http://alainet.org/active/23920\&lang=es>

ESCOBAR, Arturo (2005) El posdesarrollo como concepto y práctica social. En: Daniel Mato (coord..) Políticas de economía, ambiente y sociedad en tiempos 
de globalización. Caracas: Facultad de Ciencias Económicas y Sociales. Universidad Central de Venezuela, pp. 17-31

MAS-IPSP (2009) Bolivia país líder. Programa de Gobierno del MAS-IPSP 2010-1015, LA Paz: 156 pp.

MEDINA, Javier. (2006) Suma Qamaña. Por una convivialidad posindustrial, La Paz, Bolivia, Garza Azul Editores.

LATOUCHE. Serge (2009) "Pequeño tratado de Decrecimiento sereno". Icaria editorial.

(2003) Decrecimiento y posdesarrollo. El pensamiento creativo contra la economía del absurdo, El Viejo Topo, 2003, Latouche, Serge.

KAY, Cristóbal (2009): "Estudios rurales en América Latina en el periodo de globalización neoliberal: ¿una nueva ruralidad?, Revista Mexicana de Sociología, Vol. 71, No. 4, pp. 607-645

(2009b) Reflexiones sobre desarrollo rural y estrategias de desarrollo: Exploración de sinergias, erradicación de pobreza / Cristobal Kay. Revista Debate Agrario 44, pp. 1-23

(2007): "Enfoques sobre el Desarrollo Rural en América Latina y Europa desde Mediados del Siglo XX", PÉREZ, Edelmira [Ed.]: La Enseñanza del Desarrollo Rural: Enfoques y Perspectivas. Universidad Javeriana, Bogotá, pp. 49-111.

(2007b): "Pobreza rural en América Latina: teorías y estrategias de desarrollo", Revista Mexicana de Sociología, Vol. 69, No. 1, pp. 69-108

(1998) "¿El fin de la reforma agraria en América Latina? El legado de la reforma agraria y el asunto no resuelto de la tierra", Revista Mexicana de Sociología, vol. 60, ํo 4, pp. 61-98.

ROSTOW, W. W. (1961) Las etapas del crecimiento económico: un Manifiesto no Comunista, México: Fondo de Cultura Económica, pp. 206.

SHEANAN, Jhon, (1990), "El comercio exterior, la industrialización y el desarrollo económico". En: Modelos de desarrollo en América Latina, Pobreza, represión y estrategia económica. Alianza Editorial Mexicana, pp. 104-135

URIOSTE, Miguel; KAY, Cristóbal (2005): "Latifundios, avallasamientos y autonomías: la reforma agraria inconclusa en el Oriente", Fundación TIERRA, La Paz.

WARMAN, Arturo (1988): "El camino del poder alimentario", Historia de un bastardo. Maíz y capitalismo. Fondo de Cultura Económica, México, pp. 191214. 